\title{
The association of quantitative insulin sensitivity indices (HOMA-IR and QUICKI) with anthropometric and cardiometabolic indicators in adolescents
}

Yaser Mirzaalian¹, Mozhgan Nourian¹, Maryam Gholamalizadeh², Saeid Doaei ${ }^{3}$, Mahshid Hatami $^{4}$, Akbar Hassanzadeh ${ }^{5}$, Gholamreza Askari' ${ }^{1}$, Raheleh Farahi ${ }^{1}$

\author{
${ }^{1}$ Department of Community Nutrition, School of Nutrition and Food Sciences, Isfahan \\ University of Medical Sciences, Isfahan, Iran \\ 2Student Research Committee, Cancer Research Center, Shahid Beheshti University \\ of Medical Sciences, Tehran, Iran \\ ${ }^{3}$ Research Center of Health and Environment, Guilan University of Medical Sciences, \\ Rasht, Iran \\ ${ }^{4}$ Department of Community Nutrition, School of Nutrition and Food Sciences, Shahid \\ Beheshti University of Medical Sciences, Tehran, Iran \\ ${ }^{5}$ Department of Epidemiology and Biostatistics, School of Health, Isfahan University \\ of Medical Sciences, Isfahan, Iran
}

Submitted: 16 August 2018

Accepted: 2 February 2018

Arch Med Sci Atheroscler Dis 2019; 4: e32-e37

DOI: https://doi.org/10.5114/amsad.2019.84411

Copyright (c) 2019 Termedia \& Banach

\section{Abstract}

Introduction: Homoeostasis model assessment (HOMA-IR) and the quantitative insulin sensitivity check index (QUICKI) are used to evaluate insulin resistance. The aim of this study was to investigate the association between quantitative insulin sensitivity indices and anthropometric and cardiometabolic indicators in adolescents.

Material and methods: This descriptive-analytic cross-sectional study was conducted on 80 adolescents aged 12 to 13 years in Isfahan, Iran. Anthropometric, cardiometabolic and QUICKI and HOMA-IR indicators were measured. In the results analysis, Pearson correlation coefficient and regression analysis were used.

Results: There was a significant positive correlation between most of the anthropometric indicators and the HOMA-IR index and a significant negative correlation with QUICKI (all $p<0.0001$ ). Moreover, serum triglyceride level had a significant negative correlation with QUICKI index $(R=-0.33$, $p=0.002)$ and systolic blood pressure $(R=0.44, p<0.0001)$, and triglyceride level $(R=0.66, p<0.0001)$ had a significant positive correlation with HOMA index. The results of these two indices were highly correlated in most of the anthropometric and biochemical indices, except for the waist circumference to the neck circumference ratio and systolic blood pressure, which had a significant positive association with HOMA-IR, but did not show a significant association with QUICKI index.

Conclusions: A significant correlation between anthropometric and cardiometabolic indicators with insulin resistance indices (HOMA-IR and QUICKI) was found. Moreover, the results of these two indices were highly correlated in most of the anthropometric and biochemical indices, except for the waist circumference to the neck circumference ratio and systolic blood pressure.

Key words: QUICKI, HOMA-IR, anthropometric indices, cardiometabolic indices.

\author{
Corresponding authors: \\ Mozhgan Nourian \\ Department \\ of Community Nutrition \\ School of Nutrition \\ and Food Sciences \\ Isfahan University \\ of Medical Sciences \\ Isfahan, Iran \\ Phone: +98 3138923172 \\ E-mail: nourian@hlth.mui. \\ ac.ir \\ Saeid Doaei \\ Research Center \\ of Health and \\ Environment \\ Guilan University \\ of Medical Sciences \\ Rasht, Iran \\ Phone: +98 1333849411 \\ E-mail: sdoaei@sbmu.ac.ir
}




\section{Introduction}

Diabetes mellitus is the most common glandular disease in the world and yearly is responsible for 4 million deaths [1]. The number of people with diabetes rose from 108 million in 1980 to 422 million in 2014 [2]. Diabetes is a metabolic disease that is associated with a disorder in insulin sensitivity throughout the body. Several studies suggest that body fat distribution can play a major role in metabolic abnormalities associated with obesity, related to the strong relationship that abdominal obesity has with insulin resistance, metabolic disorders and cardiovascular disease [3-5]. Body mass index (BMI), waist measurement (WC), waist to hip ratio (WHR), waist to hip circumference, sagittal abdominal diameter and neck circumference are the most commonly used anthropometric indices for obesity monitoring [6-8].

Though some mechanisms have been proposed in the field of insulin sensitivity, more extensive studies are needed to precisely identify factors correlating with insulin resistance [9]. Although many studies have already been conducted on adult populations, there are few studies in child and adolescent populations [10]. As insulin resistance and hyperinsulinemia are found in children and adolescents before maturity, it is clear that we should seek the cause of this disorder in that course [11]. Although insulin resistance in adults has the strongest association with abdominal circumference, determination of the association between insulin resistance and anthropometric measurements at an earlier age requires further studies $[12,13]$.

Several indicators are now used for assessment of insulin sensitivity/resistance. For example, the homoeostasis model assessment-insulin resistance (HOMA-IR) index has recently been used in many studies. This index indicates insulin resistance based on the amount of fasting plasma glucose and insulin. Moreover, some recent studies suggest that the quantitative insulin sensitivity check index (QUICKI) can be a good alternative for HOMA-IR and it is believed that HOMA-IR shows insulin resistance less than the truth $[14,15]$.

Thus, according to the available studies, lack of sufficient evidence and absence of any study conducted in Iran on the association between QUICKI and HOMA-IR with anthropometric and cardiometabolic indicators in adolescents, the aim of our study was to investigate the association between insulin sensitivity indices (QUICKI and HOMA-IR) and anthropometric and cardiometabolic indicators in 12 to 13 year-old adolescents in Isfahan, Iran.

\section{Material and methods}

This cross-sectional study was conducted on 80 teenagers aged 12 to 13 years old (40 boys and
40 girls) in collaboration with the Cardiovascular Research Center of Isfahan University of Medical Sciences in Isfahan, Iran. The subjects were selected from one high school of Isfahan city by simple randomized sampling. The inclusion criteria included: age 12 to 13 years, have reached sexual maturity, and consent to participate in the study. After collecting written consent from the students and their parents, the subjects were invited to the Cardiovascular Research Center for data collection. A socio-demographic questionnaire was completed and anthropometric indices (including height, weight, BMI, waist circumference, neck circumference, and wrist circumference) were measured using the following methods. To measure the body weight, subjects were made to stand motionless on a Seca scale with minimal clothes and without shoes. The scale was calibrated to zero before each reading. Height was measured by height measuring systems (least count $0.5 \mathrm{~cm}$ ) and subjects were made to stand in a straight position, keeping heels together and without shoes. Waist circumference (WC) and neck circumference were measured in a standing position with an elastic plastic measuring tape without any pressure on it with the least count $0 / 1 \mathrm{~cm}$. The WC was measured in the middle of the lowest rib and the highest part of the pelvis [16]. The maturity of all subjects was assessed by a medical doctor.

We also collected blood samples from the participants after at least $10 \mathrm{~h}$ overnight fasting and the fasting glucose, fasting insulin, triglyceride (TG), high-density lipoprotein cholesterol (HDL-C), low-density lipoprotein cholesterol (LDL-C), TGs and total cholesterol levels were assessed. We applied a validated protocol of blood pressure measurement.

This testing provided a measurement of the serum concentrations of total cholesterol, TG, HDL-C, and calculated LDL-C. The Friedewald formula was used to calculate LDL-C from total cholesterol, triglycerides, and HDL-C. This formula calculates LDL-C by subtracting the sum of HDL-C and very low-density lipoproteins (VLDL) (triglycerides/ 5 from total cholesterol). Moreover, insulin sensitivity was calculated by the following formulas according to QUICKI and HOMA-IR [17]: QUICKI= $1 /(\log ($ fasting insulin $\mu \mathrm{U} / \mathrm{ml})+\log ($ fasting glucose $\mathrm{mg} / \mathrm{dl})$ ), HOMA-IR = (fasting insulin $\mu \mathrm{m} / \mathrm{ml}$ ) $+($ fasting glucose $\mathrm{mg} / \mathrm{dl}) / 405$.

\section{Ethical approval}

The study was approved by the Regional Ethics Committee of Isfahan University of Medical Sciences (code: IR.MUI.REC.1395.3.369).

\section{Statistical analysis}

In this study, we used SPSS software, version 20 for statistical analysis of the data. Pearson cor- 
relation coefficient and linear regression analysis were used to analyze the data. The significance level for all analysis was considered as $p<0.05$.

\section{Results}

The general characteristics of the participants are summarized in Table I. All of the participants were 12 to 13 years old. The participants had a wide range of $\mathrm{BMI}$, but the average $\mathrm{BMI}$ of the subjects was within the normal range based on the $\mathrm{WHO}$ charts for age and sex (mean BMI $=19.18 \mathrm{~kg} / \mathrm{m}^{2}$ ).

The mean systolic and diastolic blood pressure was normal (10.8 \pm 1.3 and $6.40 \pm 0.88$ respectively). Normal levels of TG $(102.85 \pm 47.64)$, total cholesterol $(153.98 \pm 31.28)$, LDL-C $(94.88 \pm 27.98)$ and HDL-C $(38.17 \pm 10.91)$ were reported by comparing the blood lipid levels with the CEP standard values.
The values of insulin resistance indices by sex are also presented in Table II. All subjects were in the normal range of QUICKI ( $>0.38$ ) and HOMA-IR $(<2.5)$ indices. The independent $t$-test indicated that the mean QUICKI in girls was higher than boys $(p=0.04)$, but the mean HOMA-IR.

\section{Association of anthropometric and biochemical indicators with insulin resistance indices}

After adjustment for sex, there were significant negative correlations between QUICKI and all of the anthropometric indicators including weight, BMI, WC, neck circumference, waist circumference to height ratio, neck circumference to height ratio, waist circumference to neck circumference ratio and neck circumference to wrist circumfer-

Table I. Mean and standard deviation of anthropometric and cardiometabolic characteristics of children participating in the study $(n=80)$

\begin{tabular}{|c|c|c|c|}
\hline \multirow[t]{2}{*}{ Variable } & \multicolumn{2}{|c|}{ Mean } & \multirow[t]{2}{*}{$P$-value } \\
\hline & Boys $(n=40)$ & Girls $(n=40)$ & \\
\hline Age [years] & 12.7500 & 12.7250 & 0.893 \\
\hline Weight [kg] & 45.0125 & 47.4375 & 0.385 \\
\hline Height $[\mathrm{cm}]$ & 1.5517 & 1.5360 & 0.359 \\
\hline $\mathrm{SBP}[\mathrm{mm} \mathrm{Hg}]$ & 10.7750 & 10.8125 & 0.899 \\
\hline $\mathrm{DBP}[\mathrm{mm} \mathrm{Hg}]$ & 6.3625 & 6.4625 & 0.615 \\
\hline Wrist circumference $[\mathrm{cm}]$ & 15.8450 & 15.6000 & 0.410 \\
\hline Waist circumference $[\mathrm{cm}]$ & 67.9075 & 70.7875 & 0.151 \\
\hline Neck circumference $[\mathrm{cm}]$ & 30.9000 & 32.4875 & 0.007 \\
\hline Triglyceride [mg/dl] & 1.0320 & 1.0250 & 0.948 \\
\hline Total cholesterol [mg/dl] & 1.4732 & 1.6062 & 0.057 \\
\hline $\mathrm{LDL}[\mathrm{mg} / \mathrm{dl}]$ & 88.6275 & 1.0112 & 0.045 \\
\hline $\mathrm{HDL}[\mathrm{mg} / \mathrm{dl}]$ & 37.4875 & 38.8500 & 0.580 \\
\hline Fasting glucose [mg/dl] & 83.6750 & 85.1250 & 0.311 \\
\hline Fasting insulin $[\mu \mathrm{U} / \mathrm{ml}]$ & 0.6225 & 0.5175 & 0.085 \\
\hline Height to wrist ratio & 9.8259 & 9.9063 & 0.609 \\
\hline Waist circumference to height ratio & 0.4375 & 0.4604 & 0.045 \\
\hline
\end{tabular}

$B M I$ - body mass index, SBP - systolic blood pressure, DBP - diastolic blood pressure, $L D L$ - low-density lipoprotein, HDL - high-density lipoprotein, QUICKI - quantitative insulin-sensitivity check index, HOMA-IR-homeostatic model assessment of insulin resistance, NS - not significant.

Table II. Comparison of QUICKI and HOMA-IR in boys and girls using $t$-test

\begin{tabular}{|lccccc|}
\hline Variable & \multicolumn{2}{c}{ Girls $(n=40)$} & \multicolumn{2}{c|}{ Boys $(n=40)$} & P-value \\
\cline { 2 - 5 } & Mean & SD & Mean & SD & \\
\hline QUICKI & 0.64 & 0.11 & 0.6 & 0.06 & 0.04 \\
\hline HOMA-IR & 0.11 & 0.05 & 0.13 & 0.06 & 0.12 \\
\hline
\end{tabular}


ence ratio (all $p<0.003$ ). Among cardiometabolic indices, TG levels had a significant negative correlation with QUICKI. The association of other cardiometabolic indicators with QUICKI were not statistically significant. Regarding anthropometric indices, there were significant positive correlations between most anthropometric indicators including weight, BMI, WC, neck circumference, waist circumference to height ratio, neck circumference to height ratio, waist circumference to neck circumference ratio and neck circumference to wrist circumference ratio with HOMA-IR index (all $p<0.0001$ ). Among cardiometabolic indicators, SBP and TG levels had positive correlations with HOMA-IR index $(p<0.0001)$, while it was not statistically significant for other cardiometabolic indicators (Table III).

By comparing QUICKI and HOMA-IR, we found that these two indices are correlated with all of the anthropometric and biochemical indicators, except for waist circumference to neck circumference ratio and systolic blood pressure, which had a significant positive correlation with HOMA-IR, but did not show a significant relationship with QUICKI.

\section{Discussion}

The results of the present study indicated significant negative correlations between QUICKI and most anthropometric indicators such as weight, BMI, WC, neck circumference, waist circumference to height ratio, and neck circumference to height ratio, and also significant positive correlations between these anthropometric indicators and HOMA-IR. Moreover, among cardiometabolic indicators, there was only a negative correlation between TG and QUICKI and a positive correlation between TG, SBP and HOMA-IR. Other cardiometabolic indicators did not correlate with insulin resistance indices.

Our results for anthropometric indicators were similar to the results of other studies. Szapary et al. [17] reported that higher values of HOMA-IR were associated with development of metabolic syndrome, obesity, and lower serum HDL levels in adolescents under 18 years old. Our study also demonstrated that individuals with higher HOMA-IR had higher weight and BMI values.

Similar findings were reported in another study performed on 691 healthy teenagers (295 with normal BMI, 205 overweight and 199 obese) indicating a significant positive correlation between HOMA-IR and BMI [18].

In the study done by De Luis et al. [19], a significant positive correlation between HOMA-IR and $B M I$ was found, and those who were obese had higher HOMA-IR levels than people with normal BMI. Moreover, in the study from Webber et

Table III. Association of anthropometric and biochemical indicators with QUICKI and HOMA-IR after adjustment for $\operatorname{sex}(n=80)$

\begin{tabular}{|c|c|c|c|c|}
\hline \multirow[t]{2}{*}{ Variable } & \multicolumn{2}{|c|}{ QUICKI } & \multicolumn{2}{|c|}{ HOMA-IR } \\
\hline & $R$ & $P$-value & $R$ & $P$-value \\
\hline Weight $[\mathrm{kg}]$ & -0.48 & 0.0001 & 0.57 & 0.0001 \\
\hline $\mathrm{BMI}\left[\mathrm{kg} / \mathrm{m}^{2}\right]$ & -0.45 & 0.0001 & 0.54 & 0.0001 \\
\hline Waist circumference $[\mathrm{cm}]$ & -0.45 & 0.0001 & 0.57 & 0.0001 \\
\hline Neck circumference $[\mathrm{cm}]$ & -0.43 & 0.0001 & 0.52 & 0.0001 \\
\hline Waist circumference to height ratio & -0.37 & 0.0001 & 0.50 & 0.0001 \\
\hline Neck circumference to height ratio & -0.32 & 0.002 & 0.45 & 0.0001 \\
\hline Waist circumference to neck circumference ratio & -0.28 & NS & 0.36 & 0.0001 \\
\hline Neck circumference to wrist circumference ratio & 0.21 & NS & -0.27 & NS \\
\hline $\mathrm{SBP}[\mathrm{mm} \mathrm{Hg}]$ & -0.27 & NS & 0.34 & 0.0001 \\
\hline $\mathrm{DBP}[\mathrm{mm} \mathrm{Hg}]$ & -0.21 & NS & 0.26 & NS \\
\hline Triglyceride [mg/dl] & -0.33 & 0.002 & 0.46 & 0.0001 \\
\hline Total cholesterol [mg/dl] & -0.19 & NS & 0.28 & NS \\
\hline LDL [mg/dl] & -0.12 & NS & 0.19 & NS \\
\hline $\mathrm{HDL}[\mathrm{mg} / \mathrm{dl}]$ & -0.21 & NS & -0.27 & NS \\
\hline
\end{tabular}

$B M I$ - body mass index, SBP - systolic blood pressure, DBP - diastolic blood pressure, $L D L$ - low-density lipoprotein, HDL - high-density lipoprotein, QUICKI - quantitative insulin-sensitivity check index, HOMA-IR - homeostatic model assessment of insulin resistance, NS - not significant. 
al. [20], HOMA-IR had a significant positive correlation with $\mathrm{BMI}$, and in subjects with $\mathrm{BMI}$ above the $85^{\text {th }}$ percentile, who were considered as overweight, HOMA-IR levels were higher than in those under the $85^{\text {th }}$ percentile, which confirms our results.

The results of a study conducted by Murdock et al. [21], to determine the effect of BMI on insulin and lipid resistance on 125 children before puberty and 122 children after puberty, a significant negative correlation between BMI and QUIC$\mathrm{KI}$ values in teenagers was observed that was in line with our results.

In another study conducted on women with gestational diabetes mellitus, HOMA-IR levels were associated with low QUICKI levels and high BMI values [22]. The findings of another study conducted on 259 healthy and obese adults and 47 healthy and obese immature adolescents to determine the insulin resistance via QUICKI indicated that patients with a history of glucose intolerance, diabetes and hypertriglyceridemia have a lower QUICKI than healthy people and also QUICKI was not significantly correlated with BMI in obese children [23]. In contrast, there was a significant negative correlation between QUICKI and $\mathrm{BMI}$ in the present study. The difference may be due to the BMI of the teenagers. This study was performed on obese adolescents, while in contrast, the BMI of our adolescents was under the $85^{\text {th }}$ percentile. It is likely that the association between QUICKI and BMI will be significant only if the overweight individuals are compared to those with normal weight. Concerning the association between HOMA-IR and QUICKI with cardiometabolic indicators, a study of 522 children (286 obese children and 236 children with normal BMI) showed that HOMA-IR was associated with systolic and diastolic blood pressure, but in our study there was no relationship between diastolic blood pressure and HOMA-IR. On the other hand, it revealed that the QUICKI in obese children was lower than in normal children. It is necessary to note that the study was conducted on children aged 6 to 15 years [24], which differed from our age range. Stagakis et al. [25], in a prospective study in patients with rheumatoid arthritis, observed a significant positive correlation between HOMA-IR levels and systolic blood pressure, which confirms the results of the present study. The present study revealed that there is a significant positive correlation between WC and HOMA-IR and a negative correlation between WC and QUICKI.

Two other studies investigating the association of insulin resistance indices demonstrated a significant negative correlation between WC and QUICKI. Similar to the results of this study, in another study, a significant positive correlation between WC and HOMA-IR levels and a significant negative correlation between and QUICKI were observed [26, 27].

In a study by Chen et al. [28], which was conducted to evaluate the level of insulin resistance in men with erectile dysfunction (ED), the QUICKI was associated with BMI. Also in this study, LDL/HDL had a significant negative correlation with the QUICKI, which was not significant in our study. Perhaps the high insulin resistance index of individuals is the reason for this mismatch, which in turn reduces NO release, endothelin level elevation and subsequently affects the LDL/HDL levels.

In another study of 99 children before puberty and 118 children after puberty, subjects with higher levels of TG/HDL had higher HOMA-IR levels and lower QUICKI [29], which were also confirmed in the present study.

In general, our study was conducted on adolescents of both genders and the relationships of both insulin resistance indices (QUICKI and HOMA-IR) with anthropometric and cardiometabolic indicators were investigated and compared. In addition to the mentioned advantage, this study has some limitations. The cross-sectional nature of the study makes it impossible to establish a cause and effect relationship between the variables. We also did not collect data on diet and physical activity, which can have a role as confounders. Our sample size was small and a longitudinal study with larger sample size is recommended in further studies to achieve transparency and certainty in the results.

In conclusion, in the present study, all anthropometric indicators including weight, BMI, WC, neck circumference, waist circumference to height ratio and neck circumference to height ratio had significant negative correlations with QUICKI and significant positive correlations with HOMA-IR. Moreover, among cardiometabolic indicators there was a significant negative correlation between TG and QUICKI whereas systolic blood pressure and TG had a significant positive correlation with $\mathrm{HO}$ MA-IR.

\section{Acknowledgments}

This work was supported by the Cardiovascular Research Center of Isfahan University of Medical Sciences, and the authors thank the personnel department. This study was extracted from an MSc dissertation which was approved by the School of Nutrition \& Food Science, Isfahan University of Medical Sciences (grant number: 3125).

\section{Conflict of interest}

The authors declare no conflict of interest. 


\section{References}

1. Ogurtsova K, da Rocha Fernandes JD, Huang Y, et al. IDF Diabetes Atlas: Global estimates for the prevalence of diabetes for 2015 and 2040. Diabetes Res Clin Pract 2017; 128: 40-50.

2. Guariguata L, Whiting DR, Hambleton I, Beagley J, Linnenkamp U, Shaw JE. Global estimates of diabetes prevalence for 2013 and projections for 2035. Diabetes Res Clin Pract 2014; 103: 137-49.

3. Skyler JS, Bakris GL, Bonifacio E, et al. Differentiation of diabetes by pathophysiology, natural history, and prognosis. Diabetes 2017; 66: 241-55.

4. Hasanvand B, Karami K, Khodadi A, Valipour M. Impact determination of strength and resistance training on Glycoside hemoglobin and blood sugar on patients with type II diabetes". Yafteh 2011; 13: 75-81.

5. Thrasher J. Pharmacologic management of type 2 diabetes mellitus: available therapies. Am J Cardiol 2017; 120 (1 Suppl): S4-16.

6. Hruschka DJ, Hadley C. How much do universal anthropometric standards bias the global monitoring of obesity and undernutrition? Obes Rev 2016; 17: 1030-9.

7. Browning LM, Hsieh SD, Ashwell M. A systematic review of waist-to-height ratio as a screening tool for the prediction of cardiovascular disease and diabetes: 0.5 could be a suitableglobal boundary value. Nutr Res Rev 2010; 23: 247-69.

8. Kalantari N, Mohammadi NK, Rafieifar S, et al. Indicator for success of obesity reduction programs in adolescents: body composition or body mass index? Evaluating a school-based health promotion project after 12 weeks of intervention. Int J Prev Med 2017; 8: 73.

9. Chan TF, Lin WT, Huang HL, et al. Consumption of sugar-sweetened beverages is associated with components of the metabolic syndrome in adolescents. Nutrients 2014; 6: 2088-103.

10. Kim B, Feldman EL. Insulin resistance as a key link for the increased risk of cognitive impairment in the metabolic syndrome. Exp Mol Med 2015; 47: e149.

11. Wu S, Gao H, Ma Y, Fu L, Zhang C, Luo X. Characterisation of betatrophin concentrations in childhood and adolescent obesity and insulin resistance. Pediatr Diabetes 2016; 17: 53-60.

12. Klein DJ, Aronson Friedman L, Harlan WR, et al. Obesity and the development of insulin resistance and impaired fasting glucose in black and white adolescent girls: a longitudinal study. Diabetes Care 2004; 27: 378-83.

13. Anhê FF, Roy D, Pilon G, et al. A polyphenol-rich cranberry extract protects from diet-induced obesity, insulin resistance and intestinal inflammation in association with increased Akkermansia spp. population in the gut microbiota of mice. Gut 2015; 64: 872-83.

14. Sarafidis PA, Lasaridis AN, Nilsson PM, et al. Validity and reproducibility of HOMA-IR, 1/HOMA-IR, QUICKI and McAuley's indices in patients with hypertension and type II diabetes. J Hum Hypertens 2007; 21: 709-16.

15. Jayagopal V, Kilpatrick E, Jennings PE, Hepburn D, Atkin S. Biological variation of homeostasis model assessment-derived insulin resistance in type 2 diabetes. Diabetes Care 2002; 25: 2022-5.

16. Geeta A, Jamaiyah H, Safiza MN, et al. Reliability, technical error of measurements and validity of instruments for nutritional status assessment of adults in Malaysia. Singapore Med J 2009; 50: 1013-8.

17. Szapary PO, Bloedon LT, Samaha FF, et al. Effects of pioglitazone on lipoproteins, inflammatory markers, and adipokines in nondiabetic patients with metabolic syn- drome. Arterioscler Thromb Vasc Biol 2006; 26: 182-8.

18. Singh Y, Garg MK, Tandon N, Marwaha RK. A study of insulin resistance by HOMA-IR and its cut-off value to identify metabolic syndrome in urban Indian adolescents. J Clin Res Pediatr Endocrinol 2013; 5: 245-51.

19. de Luis DA, Izaola O, Primo D, de la Fuente B, Aller R. Polymorphism rs3123554 in the cannabinoid receptor gene type 2 (CNR2) reveals effects on body weight and insulin resistance in obese subjects. Endocrinología, Diabetes y Nutrición 2017; 64: 440-5.

20. Webber LS, Srinivasan SR, Wattigney WA, Berenson GS. Tracking of serum lipids and lipoproteins from childhood to adulthood: the Bogalusa Heart Study. Am J Epidemiol 1991; 133: 884-99.

21. Murdock DK, Olson KJ, Juza RM, Hendricks BL. Effect of body mass index on insulin resistance and lipids in prepubertal and postpubertal children: SCHOOL observations. J Cardiometab Syndr 2006; 1: 242-7.

22. Alptekin H, Çizmecioğlu A, Işık H, Cengiz T, Yildiz M, lyisoy MS. Predicting gestational diabetes mellitus during the first trimester using anthropometric measurements and HOMA-IR. J Endocrinol Investig 2016; 39: 577-83.

23. Hřebičč J, Janout V, Malinčíková J, Horáková D, Čǐžek L. Detection of insulin resistance by simple quantitative insulin sensitivity check index QUICKI for epidemiological assessment and prevention. J Clin Endocrinol Metabol 2002; 87: 144-7.

24. Olza J, Rupérez Al, Gil-Campos M, et al. Leptin receptor gene variant rs11804091 is associated with BMI and insulin resistance in spanish female obese children: a case-control study. Int J Mol Sci 2017; 18: 1690.

25. Stagakis I, Bertsias G, Karvounaris S, et al. Anti-tumor necrosis factor therapy improves insulin resistance, beta cell function and insulin signaling in active rheumatoid arthritis patients with high insulin resistance. Arthritis Res Therapy 2012; 14: R141.

26. Landi Masquio DC, de Piano Ganen A, Munhoz da Silveira Campos R, et al. Cut-off values of waist circumference to predict metabolic syndrome in obese adolescents. Nutricion Hospitalaria 2015; 31: 1540-4.

27. Pimentel GD, Moreto F, Takahashi MM, PorteroMcLellan KC, Burini RC. Sagital abdominal diameter, but not waist circumference is strongly associated with glycemia, triacilglycerols and HDL-C levels in overweight adults. Nutricion Hospitalaria 2011; 26: 1125-9.

28. Chen S, Wu R, Huang Y, et al. Insulin resistance is an independent determinate of ED in young adult men. PLoS One 2013; 8: e83951.

29. Olson K, Hendricks B, Murdock DK. The TG to HDL ratio and its relationship to insulin resistance in pre-and postpubertal children: observation from the Wausau SCHOOL project. Cholesterol 2012; 2012: 794252. 